EPJ Web of Conferences 23, 00013 (2012)

DOI: $10.1051 /$ epjconf/20122300013

(C) Owned by the authors, published by EDP Sciences, 2012

\title{
Tunneling conductance in a system with strong electron correlation
}

\author{
J. Hong ${ }^{1,2}$ \\ ${ }^{1}$ Department of Physics, Pohang University of Science and Technology, Pohang 790-784, Korea \\ ${ }^{2}$ Asia Pacific Center for Theoretical Physics, Pohang 790-784, Korea
}

\begin{abstract}
We discuss the structure of tunneling conductance, the $d I / d V$ lineshape, observed for a system with strong electron correlation. The structure of the $d I / d V$ lineshape comprises zero-bias peak and two side peaks. We discuss the condition for compressing zero-bias peak and the origin of two side peaks. We explain why the tunneling conductance does not simply reflect the sample density of states in the tunneling experiment for a system with strong electron correlation.
\end{abstract}

Scanning tunneling spectroscopy (STS) is one of the well-known experimental skills to investigate fundamental property of a sample under consideration [1]. For normal samples, the $d I / d V$ lineshape, where $I$ and $V$ are the current and the source-drain (s-d) bias, respectively, reflects the sample density of states [2]. In this case, the electron in the scanning tunneling microscope (STM) tip can easily enter into the sample and electron tunneling depends only on the sample density of states. However, when the sample has a strong correlation, the $d I / d V$ lineshape does not reflect sample density of states. In this case, the electron in the STM tip cannot easily enter into the sample because of strong electron correlation at the site just below the tip unless the site is empty. For this reason, electron tunneling depends not only on the sample density of states but also on the local density of states at the mediating atom, which is just below the STM tip. As a result, understanding the observed $d I / d V$ lineshapes for a sample with a strong electron correlation is unsettled. Typical examples that belong to this category are quantum point contacts [3-6], a single electron transistor with a quantum dot [7-10], the STS on a magnetized atom adsorbed on a metallic surface [11-14], doped graphenes [15, 16], and high- $\mathrm{T}_{\mathrm{c}}$ superconductors [17].

We now analyze tunneling dynamics of the system with a strong electron correlation step by step. First, we consider the case of high s-d bias, i.e., $V>U$, where $U$ denotes on-site Coulomb repulsion. In this circumstance, double occupancy at the mediating atom is allowed. Hence, the effect of strong correlation is blurred by strong s-d bias. Therefore, we are not interested in this high energy dynamics. Let's consider the situation of $V \approx 0$, i.e., near zero bias. In this case, the conduction electron in the STM tip cannot tunnel into the sample by normal manner. However, an easy way for an electron in the tip to enter into the sample even at low bias exists. That is to form a singlet between the electron in the STM tip and one in the mediating atom in the sample and hop together or exchange their positions. Since position exchange does not establish net charge flow, only singlet hopping contributes to establishing tunnelling current. Therefore, the procedure, singlet hopping, changing singlet partner, and singlet hopping again, makes tunnelling current. We show the procedure in figure 1 . The process shown in figure 1 represents the Kondo dynamics in a two-reservoir Anderson impurity model near zero bias and results in the zero-bias peak in $d I / d V$ lineshape. Therefore, the zero-bias peak shown in a quantum impurity system with two reservoirs [3-6,14] corresponds to the Kondo peak.

Interestingly, the zero-bias peak is not always observed in a two-reservoir quantum impurity system under bias. There are two cases in which the zero-bias peak is substantially suppressed. One is the case when the sample has vanishing density of states at the Fermi level. High- $\mathrm{T}_{\mathrm{c}}$ superconductors [17] and bilayer graphenes may belong to this category. The other is the case when the degree of singlet coupling between the electrons in the STM tip and the mediating atom is quite weak compared with that between the electrons in the sample and the mediating atom. We do not consider the former case in this study. The latter case can be seen in the $d I / d V$ lineshape observed for a magnetized atom adsorbed on a metallic surface. We show the suppression of the zerobias peak by superimposing two lineshapes, as shown in figure 2. The $d I / d V$ lineshape showing zero-bias peak (black solid line) has been obtained for a $\mathrm{Co}$ atom adsorbed on a $\mathrm{Cu}$ (100) surface that is covered by an insulating layer $\mathrm{Cu}_{2} \mathrm{~N}$ [14]. The insulating layer suppresses fluctuations in the metallic substrate and enhances axial coherence connecting STM tip, Co atom, 
and $\mathrm{Cu}$ atom in $\mathrm{Cu}_{2} \mathrm{~N}$ [18]. Other lineshapes in figure 2 are obtained for a $\mathrm{Co}$ atom
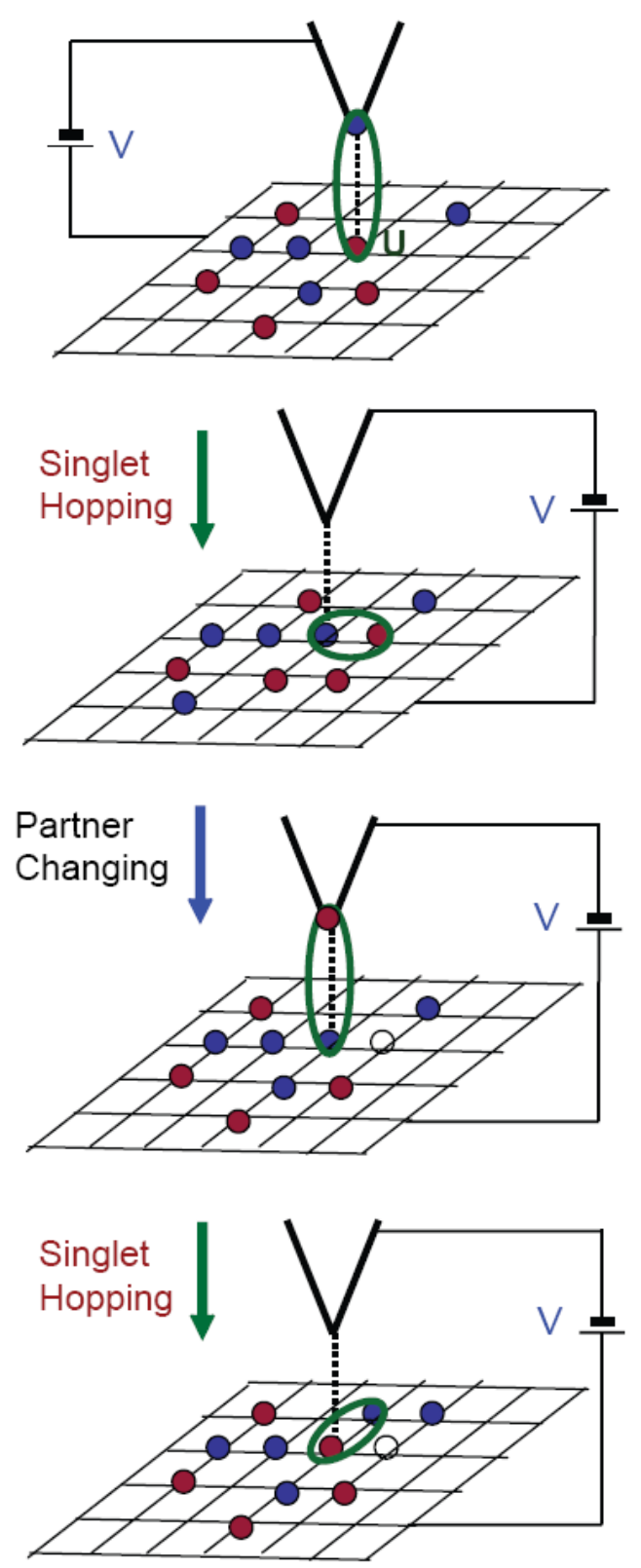

Fig. 1. Procedure of forming coherent current near zero bias. Singlet hopping, partner changing, singlet hopping, and partner changing are repeated.

adsorbed on a $\mathrm{Cu}(111)$ surface. The experimental $d I / d V$ lineshapes obtained for a $\mathrm{Cu}(100)$ surface $[13,14]$ are highly asymmetric and sample dependent. One can understand that higher fluctuation exists in $\mathrm{Cu}(100)$ surface than in $\mathrm{Cu}$ (111) surface by comparing the data obtained for $\mathrm{Cu}$ (100) substrate [13] and those for $\mathrm{Cu}$ (111) [12].

Now, we consider the bias region in which two side peaks are commonly shown in all nanocontact systems mentioned above. Figure 2 shows that two side peaks commonly appear at a finite bias in both with and without $\mathrm{Cu}_{2} \mathrm{~N}$ layer. Two side peaks are neither originated from

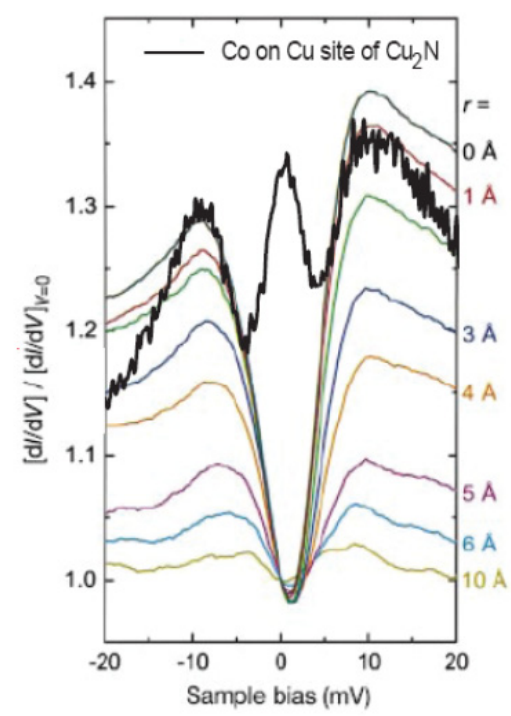

Fig. 2. Tunneling conductance measured for a Co atom adsorbed on a metallic substrate $\mathrm{Cu}(111)$ and a insulating layer $\mathrm{Cu}_{2} \mathrm{~N}$ covering $\mathrm{Cu}$ (100) (balck line).

the Coulomb peak that is formed by double occupancy at the mediating atom nor from the splitting of zero-bias peak. They are totally originated from new tunnelling mechanism. This suggests that a new tunneling scheme should exist to explain two side peaks. The origin of two side peaks have not been studied successfully until the recent study [18] by present author appears. We proved the existence of two tunnelling levels in the quantum impurity system with two reservoirs, as shown in Fig. 3. The two tunneling levels in Fig. 3 are not eigenstates of the mediating atom but the coherent tunneling levels between two reservoirs. They are provided as resonant tunneling levels connecting STM tip and the sample or two metallic reservoirs when the chemical potential of one reservoir reaches the tunneling level by applied bias. We show in the previous study [18] that a quasiparticle forming singlet performs resonant tunneling through one of these levels, which results in a side peak in the $d I / d V$ lineshape at finite bias.

Now, we discuss tunneling conductance in a system with a strong electron correlation. We employ singleimpurity Anderson model with two reservoirs. A wellknown formula for a steady current through a small interacting region connected to charge reservoirs having different chemical potentials was presented in terms of the local density of states $\rho_{d}(\omega ; V)$ at the mediating atom " $d$ " more than a decade ago $[19,20]$. The formula is written as 
$I=\frac{e}{\hbar} \int \frac{d \omega}{2} \frac{\Gamma^{L}(\omega) \Gamma^{R}(\omega)}{\Gamma^{L}(\omega)+\Gamma^{R}(\omega)}\left[f_{L}(\omega)-f_{R}(\omega)\right] \rho_{d}(\omega ; V)$,

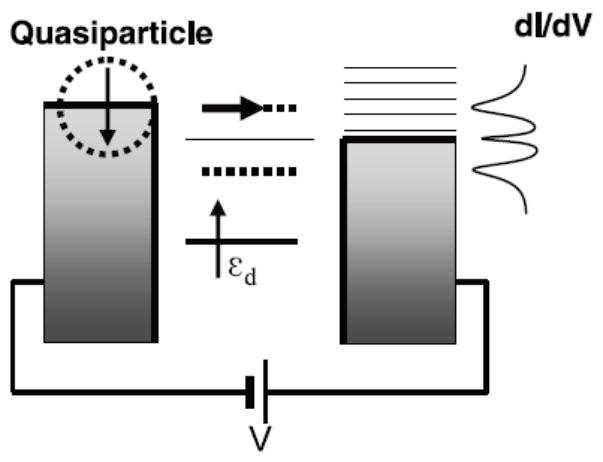

Fig. 3. Resonant tunnelling of a quasiparticle (singlet) through the coherent tunnelling level.

where $f_{L}(\omega)$ denotes the Fermi distribution for the left reservoir, for proportionate coupling functions, i.e., $\Gamma^{L}(\omega) \propto \Gamma^{R}(\omega)$. We use $\Delta=\left(\Gamma^{L}+\Gamma^{R}\right) / 4$ as energy unit. The local density of states is given by the retarded Green's function $G_{d d \sigma}^{+}(\omega)$ as $\rho_{d}(\omega ; V)=$

$-(1 / \pi) \Sigma_{\sigma} \operatorname{Im}\left[G_{d d \sigma}^{+}(\omega ; V)\right]$. Differential conductance at zero temperature is given by

$$
\frac{d I}{d V}=\left.\frac{2 e}{h} \frac{\Gamma^{L}(\omega) \Gamma^{R}(\omega)}{\Gamma^{L}(\omega)+\Gamma^{R}(\omega)} \pi \rho_{d}(\omega ; V)\right|_{\hbar \omega=e V} .
$$

We neglect the term involving $\partial \rho(\omega) / \partial V$ in Eq. (2). The left reservoir may be considered as a sample and the right reservoir as an STM tip in STS measurement. Hence, the sample density of states that appears in $\Gamma^{L}(\omega) \propto \rho_{\text {sample }}(\omega)$ dominates the $d I / d V$ lineshape when the local density of states $\left.\rho_{d}(\omega ; V)\right|_{\hbar \omega=e V}$ is flat, which occurs in a system without strong correlation. However, in a system with strong electron correlation, $\left.\rho_{d}(\omega ; V)\right|_{\hbar \omega=e V}$ dominates the $d I / d V$ lineshape.

We now discuss the effect of bias in the tunneling conductance shown in Eq. (2). In a previous study, we show that the local density of states of an up-spin electron at the Kondo impurity is given by

$\rho_{d \uparrow}(\omega)=(1 / \pi) \operatorname{Re}\left[\mathbf{M}_{\mathrm{r}}^{-1}\right]_{33}$, where

$$
\mathbf{M}_{\mathrm{r}}=\left[\begin{array}{ccccc}
-i \omega^{\prime} & -\gamma_{L L} & U_{J^{-}}^{L} & \gamma_{L R} & \gamma_{J} \\
\gamma_{L L} & -i \omega^{\prime} & U_{J^{+}}^{L} & \gamma_{J} & \gamma_{L R} \\
-U_{J^{-}}^{L^{*}} & -U_{J^{+-}}^{L^{*}} & -i \omega^{\prime} & -U_{J^{+}}^{R^{*}} & -U_{J^{-}}^{R^{*}} \\
-\gamma_{L R} & -\gamma_{J} & U_{J^{+}}^{R} & -i \omega^{\prime} & \gamma_{R R} \\
-\gamma_{J} & -\gamma_{L R} & U_{J^{-}}^{R} & -\gamma_{R R} & -i \omega^{\prime}
\end{array}\right]
$$

for the two-reservoir Anderson impurity model. Here, $\omega^{\prime}=\omega-\varepsilon_{d}-U\left\langle n_{d \downarrow}\right\rangle$, where $\varepsilon_{d}, U$, and $\left\langle n_{d \downarrow}\right\rangle$ denote the energy of the impurity state, on-site Coulomb interaction, and the average number of a down-spin electron occupying the impurity state, respectively. All the matrix elements, except the eight $U$-elements, have additional self-energy terms,

$\beta_{m n}\left[i \Sigma_{0}^{L}(\omega)+i \Sigma_{0}^{R}(\omega)\right]=\beta_{m n}\left(\Gamma^{L}+\Gamma^{R}\right) / 2$, for a flat wide

band. Detailed derivation of the matrix $\mathbf{M}_{r}$, analytic expressions of the matrix elements, and the information for $\beta_{m n}$ are given in references [18] and [21].

It is meaningful to analyze the structure of the matrix given above. The $5 \times 5$ matrix comprises two $3 \times 3$ blocks representing two single-impurity Anderson models. These two blocks commonly own the central element of the matrix that represents the single impurity of the Anderson model. The element $\gamma_{L L(R R)}$ represents the exchange and singlet hopping processes for the left (right) side and $U_{J^{ \pm}}$represents effective on-site Coulomb interaction [18]. The $2 \times 2$ corner blocks describe the mechanism of coherent tunnelling between two reservoirs. The matrix element $\gamma_{L R}\left(\gamma_{J}\right)$ represents sum (difference) of coherent tunnelings for leftward and rightward directions between two reservoirs [18]. Therefore, the bias effect appears in $\gamma_{J}$ and $\gamma_{L R}$. In equilibrium, $\gamma_{J}=0$ and under bias, $\gamma_{J}=\gamma_{L R}$. This property stems from the fact that the singlet tunnels in unidirectional manner as we have shown in Fig. 3.

If we set $\gamma_{J}=0$ in the above matrix, the spectral function $\rho_{d}(\omega ; V)$ shows only three peaks, i.e., one coherent peak at the Fermi level and two Coulomb peaks. This three-peak structure is the same as that shown in onereservoir Anderson impurity model although the spectral weight of each peak is different. The condition for unidirectional tunneling under bias, $\gamma_{J}=\gamma_{L R}$, is very important to fit experimental data. Two different conditions give rise to abrupt change in the $d I / d V$ lineshape when a bias is applied. This implies that the $d I / d V$ lineshape observed for a nanocontact system with a strong electron correlation does not have linear response regime. We show in figure 4 the typical spectral functions obtained for the condition $\gamma_{J}=0$ and $\gamma_{J}=\gamma_{L R}$. One can see that the former condition yields three peaks, while the latter yields five peaks. Three of them are coherent peaks. The coherent peak at $\omega=0$ corresponds to the dynamics described in figure 1 and two side peaks correspond to the tunneling levels shown in figure 3 . We also show that 
Kondo peak at $\omega=0$ disappears when one of $\gamma_{L L}$ and $\gamma_{R R}$ vanishes as we discussed above. Some explicit fittings to experimental data will be given in other studies of present author.

In conclusion, we discuss the reason why the $d I / d V$ lineshapes observed for a system with strong electron correlation do not simply reflect the sample density of
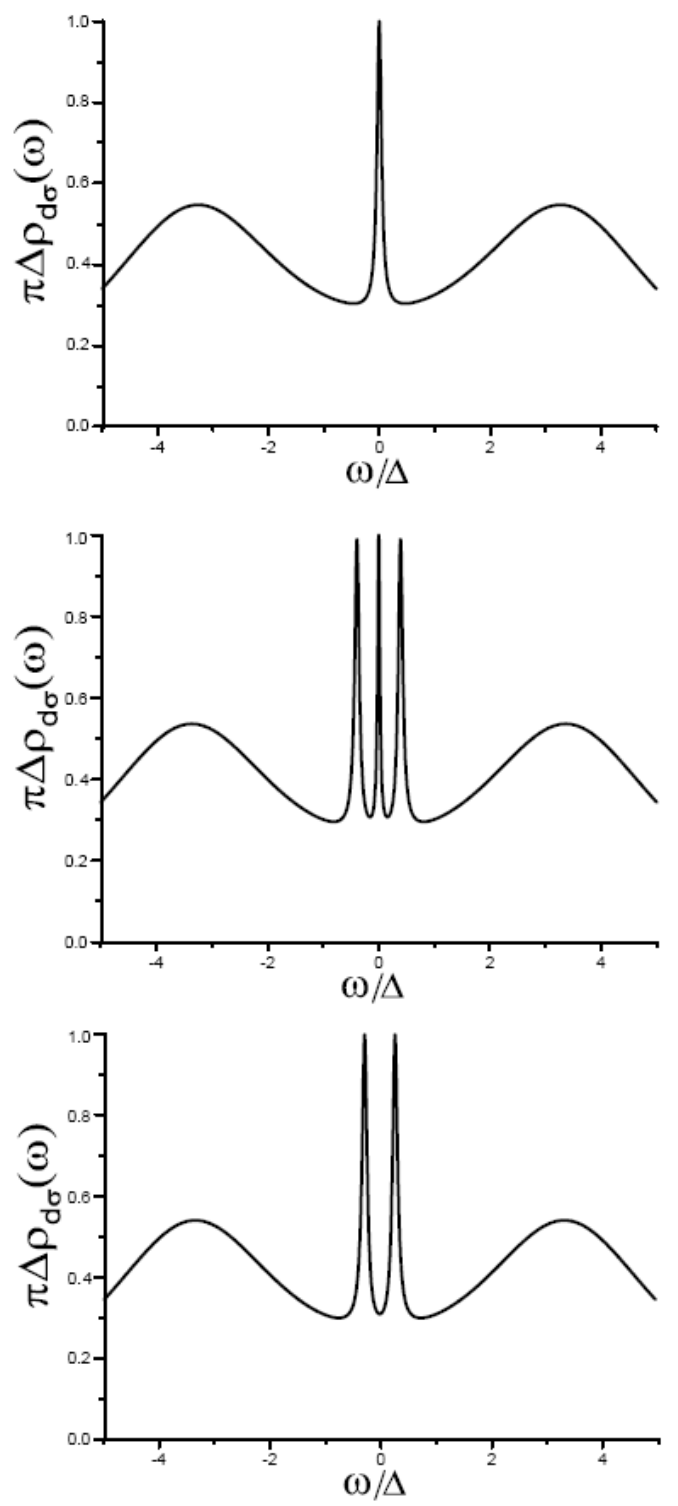

Fig. 4. Spectral functions for $\gamma_{J}=0$ (upper panel), $\gamma_{J}=\gamma_{L R}=0.4 \quad$ (mid panel) with $\gamma_{L L}=\gamma_{R R}=0.4$, $U_{J^{ \pm}}=3 / 2$, and $\beta_{m n}=0.25$. Lowermost panel is obtained by setting $\gamma_{R R}=0$ in the mid panel.

states. We also discuss the structure of the $d I / d V$ lineshapes that comprises zero-bias peak and two side peaks. We discuss the condition for compressing the zero-bias peak and the origin of two side peaks.

\section{Acknowledge}

This work was supported by the Korea Research Foundation Grant No. KRF-2008-314-C00140.

\section{References}

1. R. J. Hamers, D. F. Padowitz in Scanning Probe Microscopy and Spectroscopy edited by D. Bonnell (Wiley-VCH, Inc. New York, 2001) p. 59

2. S. H. Pan, E. W. Hudson, J. C. Davis, Appl. Phys. Lett. 73, 2992 (1998)

3. S. M. Cronenwett et al., Phys. Rev. Lett. 88, 226805 (2002)

4. L. DiCarlo et al., Phys. Rev. Lett. 97, 036810 (2006)

5. F. Sfigakis et al., Phys. Rev. Lett. 100, 026807 (2008)

6. S. Sarkozy et al., Phys. Rev. B 79, 161307(R) (2009)

7. S. M. Cronenwett, T. H. Oosterkamp, L. P. Kouwenhoven, Science 281, 540 (1998)

8. A. Kogan et al., Phys. Rev. Lett. 93, 166602 (2004)

9. S. Amasha, I. J. Gelfand, M. A. Kastner, A. Kogan Phys. Rev. B 72, 045308 (2005)

10. T. S. Jespersen et al., Phys. Rev. B 74, 233304 (2006)

11. V. Madhavan et al., Science 280, 567 (1998)

12. H. C. Manoharan, C. P. Lutz, D. M. Eigler, Nature 403, 512 (2000)

13. N. Neel et al., Phys. Rev. Lett. 98, 016801 (2007)

14. T. Choi T, C. D. Ruggiero, J. A. Gupta, J. Vac. Sci. Technol. B 27, 887 (2009)

15. Y. Zhang et al., Nat. Phys. 4, 627 (2008)

16. V. W. Brar et al., Phys. Rev. Lett. 104, 036805 (2010)

17. O. Fischer, M. Kugler, I. Maggi-Aprile, C. Berthod, C. Renner, Rev. Mod. Phys. 79, 353 (2007)

18. J. Hong, J. Phys.: Condens. Matter 23, 275602 (2011)

19. Y. Meir, N. S. Wingreen, Phys. Rev. Lett. 68, 2512 (1992)

20. S. Hershfield, J. H. Davies, J. W. Wilkins, Phys. Rev. B 46, 7046 (1992)

21. J. Hong, J. Phys.: Condens. Matter 23, 225601 (2011) 